\title{
Ropivacaine vs. levobupivacaine: Analgesic effect of combined spinal-epidural anesthesia during childbirth and effects on neonatal Apgar scores, as well as maternal vital signs
}

\author{
QIUJU CHENG ${ }^{1}$, WEIQIANG ZHANG ${ }^{2}$, YANLING LU ${ }^{2}$, JINHAI CHEN ${ }^{1}$ and HANG TIAN ${ }^{1}$ \\ Departments of ${ }^{1}$ Anesthesiology, and ${ }^{2}$ Gynecology and Obstetrics, \\ Guangzhou Women and Children's Medical Center, Guangzhou, Guangdong 510623, P.R. China
}

Received September 14, 2018; Accepted April 5, 2019

DOI: $10.3892 / \mathrm{etm} .2019 .7776$

\begin{abstract}
The present study aimed to investigate and compare the analgesic effect and safety of ropivacaine or levobupivacaine in combined spinal-epidural anesthesia during childbirth and their effects on neonatal Apgar scores, as well as maternal and neonatal vital signs. A total of 615 maternal patients undergoing labor between April 2016 and March 2017 were divided into two groups according to the analgesic used for combined spinal-epidural anesthesia during childbirth: The ropivacaine group $(n=318)$ and the levobupivacaine group $(n=297)$. The onset time of analgesia in the two groups was determined and the pain score on the visual analog scale was assessed at the time of delivery (T3). At pre-analgesia, 30 min after analgesia (T2), at T3 and during maternal wound suturing (T4), the systolic blood pressure (SBP), diastolic blood pressure (DBP) and heart rate (HR) were assessed. The cesarean section rate, neonatal 1- and 5-min Apgar scores and neonatal asphyxia at T4 were also determined. The onset time of analgesia in the ropivacaine group was significantly reduced compared with that in the levobupivacaine group $(\mathrm{P}<0.05)$. At T2 and T4, the SBP was significantly higher in the levobupivacaine group than that in the ropivacaine group $(\mathrm{P}<0.05)$. At T2, T3 and T4, the DBP was significantly lower in the levobupivacaine group compared with those in the ropivacaine group $(\mathrm{P}<0.05)$. At $\mathrm{T} 2$, the HR was significantly lower in the levobupivacaine group than that in the ropivacaine group $(\mathrm{P}<0.05)$. The cesarean section rate was significantly lower in the ropivacaine group compared with that in the levobupivacaine group [4.09\% ( $\mathrm{n}=13)$ vs. $22.89 \%(\mathrm{n}=68) ; \mathrm{P}<0.01]$. In conclusion, the use of combined spinal-epidural anesthesia with ropivacaine or levobupivacaine has an excellent analgesic
\end{abstract}

Correspondence to: Dr Hang Tian, Department of Anesthesiology, Guangzhou Women and Children's Medical Center, 9 Jinsui Road, Tianhe, Guangzhou, Guangdong 510623, P.R. China

E-mail: hangtian5434664@163.com

Key words: ropivacaine, levobupivacaine, anesthesia, delivery, analgesia effect during childbirth. However, compared with levobupivacaine, ropivacaine for labor analgesia had a faster onset and a lesser impact on maternal vital signs, and was associated with a reduced maternal cesarean section rate among patients who did not opt for cesarean section in the beginning; therefore, it is useful in clinical practice.

\section{Introduction}

Pain is an unavoidable physiological process that mothers experience during natural childbirth (1). According to the Finnerup pain score (2), the highest pain level occurs during delivery. In clinical practice, a vaginal delivery is preferred, but for patients who cannot tolerate severe pain or who encounter complications that threaten the mother's or baby's survival, a cesarean delivery may be performed (3). At present, $42.35 \%$ of deliveries worldwide are performed by cesarean section, and in certain developed countries and regions, the cesarean section rate is even higher (4). Huang et al (5) indicated that the cesarean section rate has increased every year since 2010 due to a large proportion of females being unable to overcome their inner fear of labor pain during childbirth. However, Mylonas and Friese (6) determined that the average recovery period from a cesarean section was 3-5 weeks longer than recovery from a vaginal delivery, and since the neonate does not pass through the birth canal during the cesarean section, their lungs and nerve endings are not stimulated. Therefore, cesarean delivery may affect the newborn's growth and development.

Clinical application of anesthesia during labor and delivery to reduce the negative impact of pain is common. The most frequently used method is combined spinal-epidural anesthesia. Under local anesthesia of the maternal lumbar nerves and below, the patient's mind remains awake, supporting the normal delivery process (7).

The most commonly used drug for traditional combined spinal-epidural anesthesia is levobupivacaine. Levobupivacaine is safer than bupivacaine and is less toxic to the central nervous system and heart. However, in recent years, continuous studies have indicated that females experienced a certain degree of cardiac dysfunction after the application of levobupivacaine in combined spinal-epidural anesthesia. Neonatal nerves may also be damaged by the use of anesthetic drugs used for cesarean 
section (8). At present, with the increasing cesarean section rate in clinical practice, research is urgently required in order to determine how to reduce the impact of anesthetic drugs on mothers and neonates during cesarean deliveries. There have been advances in research and medical technology in recent years (9-11), from which the application of ropivacaine has evolved. Ropivacaine is a recently developed local anesthetic drug. It is suitable for anesthesia during maternal delivery and has little impact on the uterus, placenta and maternal hemodynamics (12). Kathuria et al (13) reported that the use of combined spinal-epidural anesthesia with ropivacaine greatly reduces adverse reactions in maternal females, and has a better effect than traditional anesthetic drugs. In 2016, combined spinal-epidural anesthesia with ropivacaine has been introduced for application during the delivery process.

In the present study, the use of combined spinal-epidural anesthesia with ropivacaine for deliveries at our hospital since 2016 was retrospectively analyzed. The present analysis aimed to validate that the use of combined spinal-epidural anesthesia with ropivacaine is beneficial regarding the safety of mothers and neonates, provide a useful reference for clinical use and promote the use of ropivacaine in combined spinal-epidural anesthesia.

\section{Materials and methods}

General information. A retrospective analysis of 615 maternal patients who delivered between April 2016 and March 2017 at Guangzhou Women and Children's Medical Center (Guangzhou, China) was performed. The inclusion criteria were as follows: Full-term pregnancy, no contraindications for intraspinal anesthesia, use of combined spinal-epidural anesthesia with ropivacaine or levobupivacaine, vertex presentation of a singleton pregnancy and the availability of complete medical records. Based on these criteria, 942 patients were considered for inclusion in the study. The following exclusion criteria were then applied: Cardiovascular or cerebrovascular disease $(n=78)$, failure of major organs $(n=16)$, drug allergies $(n=31)$, severe eclampsia $(n=14)$, tumors $(n=33)$, physical disability $(n=90)$, family history of disease $(n=54)$ or transfer to another facility $(n=11)$. Ultimately, 615 patients were included in the study. All of these patients provided written informed consent. The patients were divided into two groups based on the drugs used during delivery: The ropivacaine group (combined spinal-epidural anesthesia with ropivacaine) included 318 cases and the levobupivacaine group (combined spinal-epidural anesthesia with levobupivacaine) included 297 cases. Each procedure was approved by the Ethics Committee of the Guangzhou Women and Children's Medical Center (Guangzhou, China) and conformed with the Intrapartum Care: Care of Healthy Women and Their Babies During Childbirth guidelines (14) of the National Collaborating Centre for Women's and Children's Health.

Anesthetic method. The patients underwent dural puncture through the L2-3 space when the cervix was dilated by $3-4 \mathrm{~cm}$. The catheter was inserted $3 \mathrm{~cm}$, after which ropivacaine or levobupivacaine was injected $(4 \mathrm{ml}$ each; concentration, $0.125 \%$ ) with micro-pump. After $10 \mathrm{~min}, 4 \mathrm{ml}$ ropivacaine or $4 \mathrm{ml}$ levobupivacaine containing $2 \mu \mathrm{g} / \mathrm{ml}$ sufentanil were again injected, and this was repeated a total of 3 times at 30 -min intervals. The amount of controlled analgesia was set to $4 \mathrm{ml}$ and the time was set to $15 \mathrm{~min}$. After delivery, the micro-pump was turned off. If the maternal patient was still unable to give birth normally after anesthesia, a cesarean section was performed.

Parameters observed. The clinical data collected were age, weight and gestational age. The onset of analgesia was measured from the initial injection of the drug to the disappearance of pain in the patient. A visual analog pain scale (15) was used to assess the maternal patient's pain at T3, with 0 representing no pain and 10 representing unbearable pain. Systolic blood pressure (SBP), diastolic blood pressure (DBP) and heart rate (HR) were measured at pre-analgesia (T1), 30 min after analgesia (T2), at the time of delivery (T3) and at the time of wound suturing (T4). The cesarean section rate, neonatal 1- and 5-min Apgar scores (14), and neonatal asphyxia at $\mathrm{T} 4$, as well as the incidence of post-dural puncture headache (PDPH) after anesthesia was also analyzed in the two groups.

Statistical analysis. SPSS version 22.0 statistical software (IBM Corp.) was used to analyze and process the data. Enumeration data, including patient age and analgesia onset time, were expressed as the mean \pm standard deviation, and the t-test was used for comparisons between the two groups. Measurement data, including cesarean section and neonatal asphyxia, were expressed as rates. The Chi-squared test was used for group comparisons. Repeated-measures analysis of variance with Bonferroni's post-hoc test was used for comparison among multiple time-points. $\mathrm{P}<0.05$ was considered to indicate a statistically significant difference.

\section{Results}

Comparison of clinical data between the two groups. As presented in Table I, there was no significant difference in age, maternal body weight, gestational age or blood test results between the two groups $(\mathrm{P}>0.05)$. Thus, the two groups were comparable, ensuring reliable results.

Comparison of analgesia onset time and pain scores between the two groups. The onset time of analgesia in the ropivacaine group $(3.84 \pm 0.54 \mathrm{~min})$ was significantly faster than that in the levobupivacaine group $(6.94 \pm 1.04 \mathrm{~min} ; \mathrm{P}<0.05)$. There was no significant difference in pain scores between the two groups (P>0.05; Figs. 1 and 2; Table II).

Comparison of vital signs of maternal patients between the two groups. There was no significant difference in SBP between the two groups at T1 (P>0.05). The SBP was significantly higher in the levobupivacaine group compared with that in the ropivacaine group at T2 and T4 $(\mathrm{P}<0.05)$, while the SBP was significantly lower in the levobupivacaine group compared with that in the ropivacaine group at $\mathrm{T} 3(\mathrm{P}<0.05)$. Observation of the trend of SBP in the two groups indicates that from the start of analgesia, SBP remained stable in the ropivacaine group from $\mathrm{T} 2$ but steadily decreased in the levobupivacaine group 
Table I. Comparison of clinical data between two groups of maternal patients.

\begin{tabular}{|c|c|c|c|c|}
\hline Parameter & Ropivacaine group $(n=318)$ & Levobupivacine group $(\mathrm{n}=297)$ & $\operatorname{tor} \chi^{2}$ & P-value \\
\hline Age (years) & $28.53 \pm 4.27$ & $28.04 \pm 5.15$ & 1.29 & 0.20 \\
\hline BMI (kg) & $22.16 \pm 2.84$ & $21.96 \pm 3.16$ & 0.83 & 0.41 \\
\hline Gestational week & $38.54 \pm 2.04$ & $38.86 \pm 2.59$ & 1.71 & 0.09 \\
\hline White blood cell count $\left(\mathrm{x} 10^{9} / 1\right)$ & $12.08 \pm 5.07$ & $12.53 \pm 5.24$ & 1.08 & 0.28 \\
\hline Red blood cell count (x10 $12 / 1)$ & $7.88 \pm 2.67$ & $7.53 \pm 3.74$ & 1.34 & 0.18 \\
\hline Platelet count $\left(\times 10^{9} / 1\right)$ & $124.85 \pm 26.53$ & $128.17 \pm 27.02$ & 1.54 & 0.12 \\
\hline Primipara & & & 1.28 & 0.26 \\
\hline Yes & $227(71.38)$ & $224(75.42)$ & & \\
\hline No & $91(28.62)$ & $73(24.58)$ & & \\
\hline
\end{tabular}

Values are expressed as the mean \pm standard deviation or $\mathrm{n}(\%)$. BMI, body mass index.

Table II. Onset time and pain score of analgesic drugs in the two groups.

\begin{tabular}{lcrr}
\hline Parameter & Ropivacaine group (n=318) & Levobupivacine group (n=297) & $\mathrm{t}$ \\
\hline Onset time (min) & $3.84 \pm 0.54$ & $6.94 \pm 1.04$ & 48.83 \\
Pain score at T3 & $2.99 \pm 0.45$ & $3.04 \pm 0.51$ & $<0.01$ \\
\hline
\end{tabular}

Values are expressed as the mean \pm standard deviation. T3, the time of delivery.

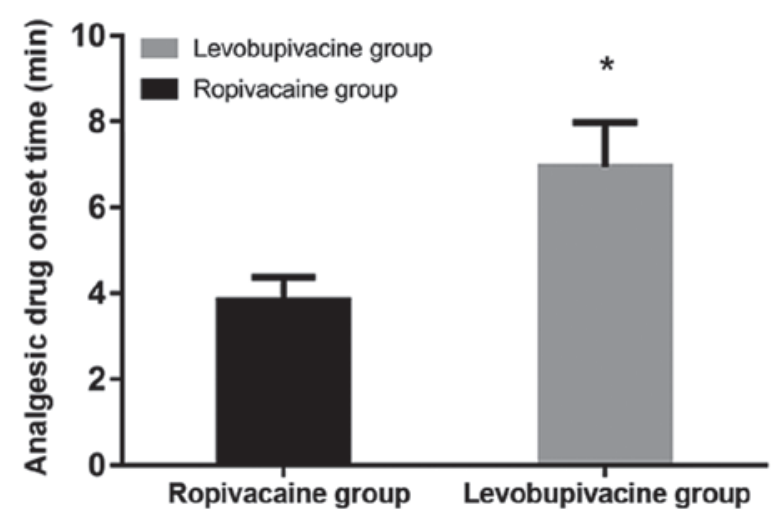

Figure 1. Onset time of analgesia in the two groups. The onset time of analgesia was significantly shorter in the ropivacaine group than that in the levobupivacaine group. ${ }^{*} \mathrm{P}<0.05$ vs. ropivacaine group.

until delivery and then slightly increased at the time-point of suturing (Fig. 3; Table III).

There was no significant difference in DBP between the two groups at $\mathrm{T} 1(\mathrm{P}>0.05)$. Regarding the trend of DBP changes in the two groups, it remained stable in the ropivacaine group with no significant change, while the DBP in the levobupivacaine group exhibited more fluctuations (Fig. 4; Table III).

There was no significant difference in HR between the two groups at $\mathrm{T} 1 \quad(\mathrm{P}>0.05)$. The HR was significantly lower in the levobupivacaine group compared with that in the ropivacaine group at $\mathrm{T} 2(\mathrm{P}<0.05)$, but the HR in the levobupivacaine group was significantly higher than that in the ropivacaine group at T3 and T4 $(\mathrm{P}<0.05)$. Regarding the trend of HR changes in the two groups, the ropivacaine group maintained a stable

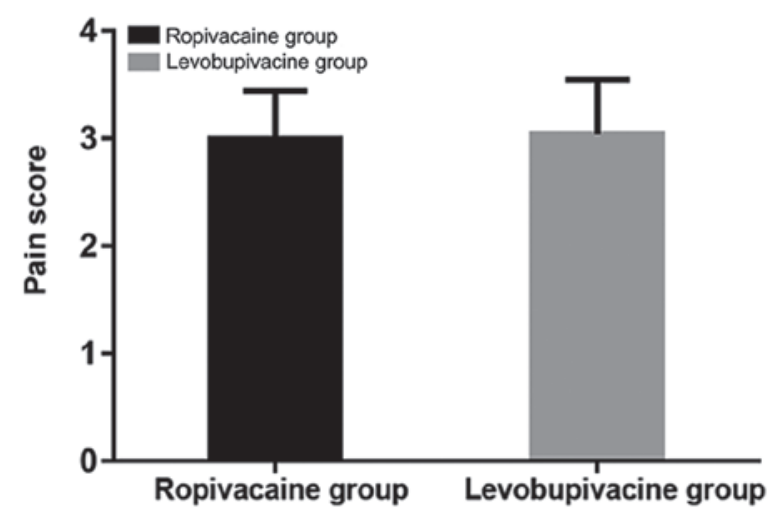

Figure 2. Visual analog scale score of analgesia in the two groups at the time of delivery. There was no significant difference in the pain scores between the two groups $(\mathrm{P}>0.05)$.

downward trend after analgesia, while in the levobupivacaine group, the HR increased after the onset of analgesia, while the ropivacaine group exhibited a steady downward trend (Fig. 5; Table III).

Comparison of delivery method and neonates. The cesarean section rate was significantly lower in the ropivacaine group $(n=13,4.09 \%)$ compared with that in the levobupivacaine group $(\mathrm{n}=68,22.89 \% ; \mathrm{P}<0.01)$. The neonatal asphyxia rate was not significantly different between the ropivacaine group $(0.63 \%)$ and the levobupivacaine group (1.01\%; $\mathrm{P}=0.60)$. There was no significant difference in the 1- and 5-min Apgar scores between the two groups $(\mathrm{P}>0.05)$. The incidence of $\mathrm{PDPH}$ in the ropivacaine group was $17.61 \%$, and that in the 
Table III. Comparison of maternal vital signs between the two groups at different time-points.

\begin{tabular}{lcccccc}
\hline Parameter & T1 & T2 & T3 & T4 & F & P-value \\
\hline SBP (mmHg) & & & & & & \\
$\quad$ Ropivacaine group & $135.97 \pm 5.67$ & $126.07 \pm 5.17^{\mathrm{a}}$ & $125.94 \pm 4.34^{\mathrm{a}}$ & $126.01 \pm 4.15^{\mathrm{a}}$ & 334.82 & $<0.01$ \\
Levobupivacine group & $136.24 \pm 5.72$ & $128.64 \pm 5.47^{\mathrm{a}, \mathrm{b}}$ & $123.86 \pm 4.02^{\mathrm{a}-\mathrm{c}}$ & $127.59 \pm 4.26^{\mathrm{a}-\mathrm{d}}$ & 331.34 & $<0.01$ \\
DBP (mmHg) & & & & & & \\
$\quad$ Ropivacaine group & $84.92 \pm 4.28$ & $82.16 \pm 5.04$ & $84.86 \pm 4.42$ & $84.37 \pm 4.65$ & 1.05 & 0.37 \\
Levobupivacine group & $85.04 \pm 4.68$ & $84.57 \pm 4.52^{\mathrm{a}, \mathrm{b}}$ & $87.53 \pm 4.88^{\mathrm{a}-\mathrm{c}}$ & $79.54 \pm 3.83^{\mathrm{a}-\mathrm{d}}$ & 166.51 & $<0.01$ \\
HR (bpm) & & & & & & \\
Ropivacaine group & $88.64 \pm 13.27$ & $84.17 \pm 12.30^{\mathrm{a}}$ & $80.24 \pm 13.86^{\mathrm{a}}$ & $76.34 \pm 12.53^{\mathrm{a}}$ & 52.12 & $<0.01$ \\
Levobupivacine group & $88.29 \pm 14.13$ & $82.54 \pm 14.86^{\mathrm{a}, \mathrm{b}}$ & $89.62 \pm 13.42^{\mathrm{a}, \mathrm{b}}$ & $92.53 \pm 13.65^{\mathrm{a}-\mathrm{d}}$ & 26.57 & $<0.01$ \\
\hline
\end{tabular}

${ }^{\text {a }} \mathrm{P}<0.05$ compared with the same group at $\mathrm{T} 1 ;{ }^{\mathrm{b}} \mathrm{P}<0.05$ compared with ropivacaine group at $\mathrm{T} 2 ;{ }^{\mathrm{c}} \mathrm{P}<0.05$ compared with ropivacaine group at $\mathrm{T} 3$; ${ }^{\mathrm{d}} \mathrm{P}<0.05$ compared with ropivacaine group at $\mathrm{T} 4$. Values are expressed as the mean \pm standard deviation. Time-points: T1, pre-analgesia; $\mathrm{T} 2,30$ min after analgesia; T3, at the time of delivery; T4, during maternal wound suturing. SBP, systolic blood pressure; DBP, diastolic blood pressure; HR, heart rate; bpm, beats per minute.

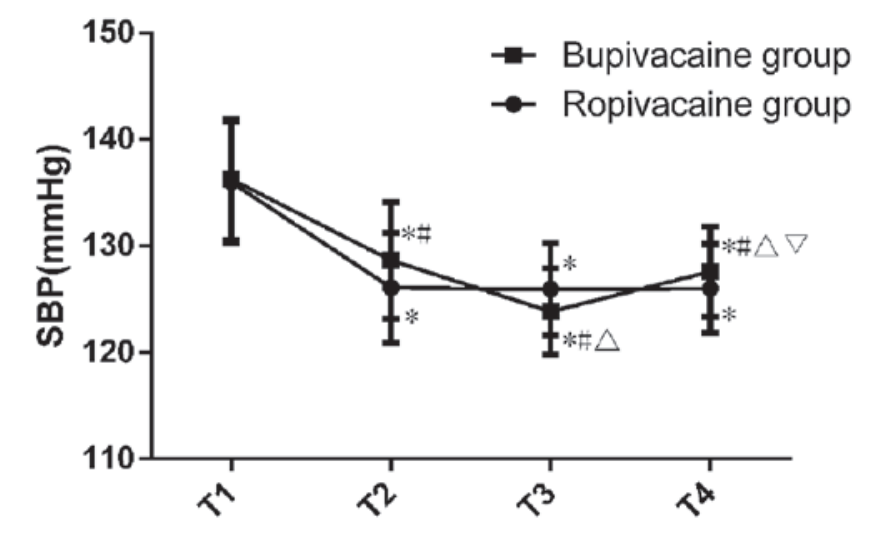

Figure 3. SBP in the two groups at different time-points. In the ropivacaine group, SBP decreased from the onset of analgesia until delivery, while the SBP in the levobupivacaine group increased significantly after analgesia ${ }^{*} \mathrm{P}<0.05$ vs. $\mathrm{T} 1$ within the same group; ${ }^{\text {}} \mathrm{P}<0.05$ vs. the ropivacaine group at $\mathrm{T} 2 ;{ }^{\Delta} \mathrm{P}<0.05$ vs. ropivacaine group at $\mathrm{T} 3 ;{ }^{\triangleright} \mathrm{P}<0.05$ vs. ropivacaine group at $\mathrm{T} 4$. Time-points: T1, pre-analgesia; T2, 30 min after analgesia; T3, at the time of delivery; T4, during maternal wound suturing. SBP, systolic blood pressure.

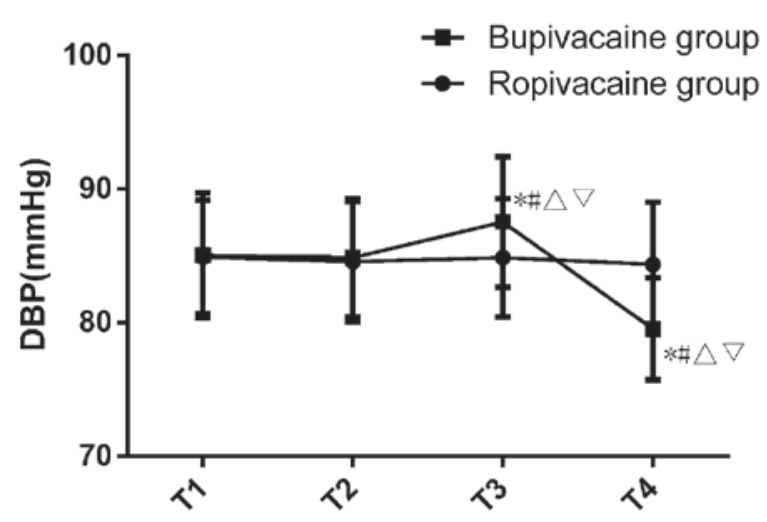

Figure 4. DBP in the two groups at different time-points. The ropivacaine group remained stable with no significant changes, while the levobupivacaine group exhibited large fluctuations. ${ }^{*}<0.05$ vs. T1 within the same group; ${ }^{\text {"}} \mathrm{P}<0.05$ vs. the ropivacaine group at $\mathrm{T} 2 ;{ }^{\Delta} \mathrm{P}<0.05$ vs. ropivacaine group at $\mathrm{T} 3 ;{ }^{\circ} \mathrm{P}<0.05$ vs. ropivacaine group at $\mathrm{T} 4$. Time-points: $\mathrm{T} 1$, pre-analgesia; $\mathrm{T} 2,30$ min after analgesia; $\mathrm{T} 3$, at the time of delivery; T4, during maternal wound suturing. DBP, diastolic blood pressure.

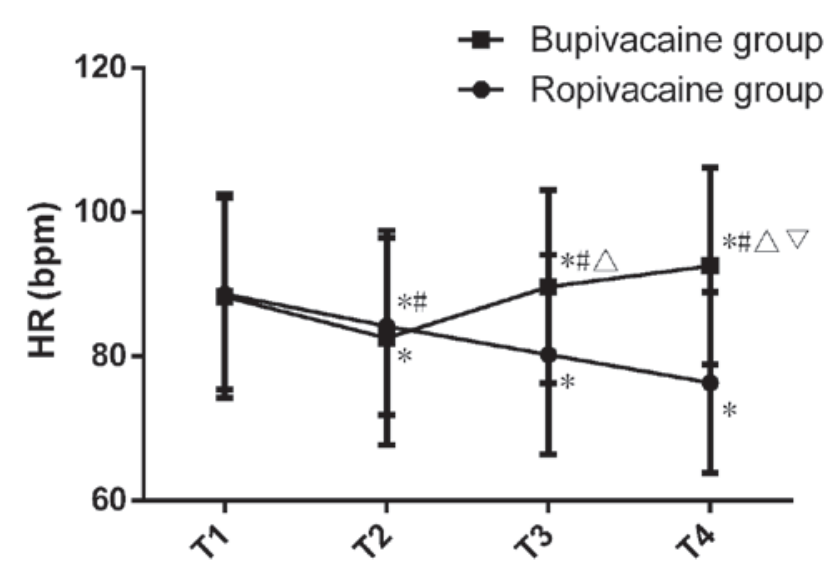

Figure 5. HR in the two groups at different time-points. The ropivacaine group exhibited a stable decline in HR after analgesia, while the levobupivacaine group had more obvious HR fluctuations. Time-points: T1, pre-analgesia; T2, 30 min after analgesia; T3, at the time of delivery; T4, during maternal wound suturing. HR, heart rate; bpm, beats per minute. ${ }^{*} \mathrm{P}<0.05$ vs. T1 within the same group; ${ }^{\#} \mathrm{P}<0.05$ vs. the ropivacaine group at $\mathrm{T} 2 ;{ }^{\circ} \mathrm{P}<0.05$ vs. ropivacaine group at $\mathrm{T} 3 ;{ }^{\triangleright} \mathrm{P}<0.05$ vs. ropivacaine group at $\mathrm{T} 4$.

levobupivacaine group was $20.88 \%$; the difference was not statistically significant $(\mathrm{P}>0.05$; Table IV).

\section{Discussion}

During the vaginal delivery process, the fetus passes through the uterus and narrow vagina, and paroxysmal contractions and friction from the movement of the fetus stimulate the mother's nerve endings and produce nerve impulses that pass from the lumbar plexus to the brain, producing a sense of severe pain during the delivery process $(16,17)$. Delivery does not only cause pain for the mother, but is also associated with negative emotions, including anxiety and fear. These also affect the vaginal delivery success rate $(18,19)$. Cesarean section may effectively reduce pain during childbirth, but this traumatic surgical procedure causes greater damage and requires a longer recovery time (20). During cesarean sections, major 
Table IV. Comparison of pregnancy outcomes between the two groups of patients.

\begin{tabular}{lcrr}
\hline Item & Ropivacaine group (n=318) & Levobupivacine group (n=297) & $\chi^{2}$ or t \\
\hline Cesarean section rate & $13(4.09)$ & $68(22.89)$ & 54.34 \\
Neonatal asphyxia rate & $2(0.63)$ & $3(1.01)$ & 0.28 \\
Neonatal Apgar score & & & 0.60 \\
At 1 min & $9.82 \pm 0.42$ & $9.78 \pm 0.51$ & 1.07 \\
At 5 min & $9.12 \pm 0.84$ & $9.23 \pm 0.73$ & 0.29 \\
PDPH incidence & $56(17.61)$ & $62(20.88)$ & 0.08 \\
\hline
\end{tabular}

Values are expressed as the mean \pm standard deviation or $\mathrm{n}(\%)$. PDPH, post-dural puncture headache.

bleeding may occur, which may cause secondary damage to other organs in the abdominal cavity, and urinary tract infections or endometriosis may develop after surgery (21). Furthermore, the fetus does not experience extrusion through the birth canal or exposure to its bacteria, resulting in poorer natural immunity than for infants born vaginally. In clinical practice, vaginal delivery is recommended if possible (22). Reduction of pain during labor and vaginal delivery is the focus of current clinical research. The most common safe and effective method in clinical practice is to perform combined spinal-epidural anesthesia during labor and to reduce pain by continuous or intermittent injection of local anesthetic drugs through an intraspinal catheter (23). Furthermore, as anesthetic drugs are frequently accompanied by adverse reactions during labor, the clinical dosage of combined spinal-epidural anesthesia requires to be strictly controlled, and maternal females still experience changes in their contractions during delivery. At present, ropivacaine and levobupivacaine are the most common analgesic drugs. However, the value of these two analgesic drugs for labor and delivery remains controversial. Therefore, the present study provides a reference for the use of analgesic drugs during childbirth that may be used in future clinical trials.

The results of the present retrospective study indicated that the group receiving combined spinal-epidural anesthesia with ropivacaine achieved analgesia faster than the group receiving combined spinal-epidural anesthesia with levobupivacaine. Compared with levobupivacaine, ropivacaine had a faster analgesic onset time and was more beneficial, with fewer side effects for the mother and a reduced rate of cesarean section.

Through the combined spinal-epidural anesthesia, the maternal labor pain, fear during childbirth and postpartum fatigue are greatly reduced, so that the patient may be delivered naturally. A study by Wang et al (24) indicated that nerve block anesthesia affects the mother's ability to actively hold her breath and the pelvic floor muscle tension is reduced, affecting the internal rotation and head flexion of the fetus. Therefore, the probability of the fetus becoming transverse or remaining in an occipitoposterior position is increased. The length of labor is prolonged and the rate of cesarean section naturally increases. In the present study, the cesarean section rate was $24.24 \%$ in the levobupivacaine group, which validates the above point of view. Ropivacaine is less toxic to the heart than levobupivacaine, and it has a lighter neuromotor block effect (25). Combined spinal-epidural anesthesia with ropivacaine has a lesser influence on the cervical and vaginal motor nerves in the lower uterine segment, which decreases the birth canal resistance. The cervix opens and the fetal head descends, thus significantly increasing the success rate of vaginal delivery. In the present study, the cesarean section rate in the ropivacaine group was only $4.09 \%$. Ropivacaine has strong reactivity with $\alpha 1$-acid glycoprotein in the human body (26), which may further ensure fetal safety and reduce the need for surgery. This is also consistent with the results of Xiao et al (27). Ropivacaine is a long-acting amide local anesthetic with a stronger ability to inhibit nerve conduction in pain-sensing fibers, but it has a lesser effect on the central nervous system and the cardiovascular system (18). Therefore, low-concentration medications not only provide effective analgesia but also affect other system functions to a lesser extent. In the present study, SBP, DBP and HR all remained stable after analgesia in the ropivacaine group and were significantly better than those in the levobupivacaine group. There was no significant difference in the neonatal asphyxia rate or Apgar scores between the two groups, and the values were in accordance with the normal rates and scores, which indicated that neither drug affected the fetus. Thus, the two drugs have good applicability. Regarding the incidence of PDPH after anesthesia in the two groups, there was no significant difference between the ropivacaine group and the levobupivacaine group, suggesting that there is no significant difference between the effects of the two anesthetic drugs on the mother. The two drugs are suitable for anesthesia for delivery.

In summary, the use of combined spinal-epidural anesthesia with ropivacaine or levobupivacaine had an excellent analgesic effect and improved safety for childbirth. However, compared with levobupivacaine, ropivacaine has a faster effect of labor analgesia and a lesser impact on maternal vital signs, and effectively reduced the rate of cesarean section; therefore, it is worth promoting its clinical use.

However, there were certain limitations to the present study, including the relatively young population. Ropivacaine intolerance may occur in mothers $>30$ years old, since ropivacaine has toxic effects on the cardiovascular system. When the blood concentration is too high, cardiac conduction and myocardial contractility may be inhibited. The bodily functions, including 
blood flow, immunity and rehabilitation ability, of older pregnant females are generally worse than those of the cohort of the present study. Therefore, longer-term follow-up surveys on the subjects of the present study will be performed with continuous refinement and improvement of the assessments and analyses.

\section{Acknowledgements}

Not applicable.

\section{Funding}

This work was supported by the Science and Technology Program of Guangzhou, China (grant no. 201709010097).

\section{Availability of data and materials}

The datasets used and/or analyzed during the current study are available from the corresponding author on reasonable request.

\section{Authors' contributions}

QC conceived and designed the study and interpreted results. WZ and JC contributed to the design of the study and the interpretation of the experimental results. YL and HT performed experiments, analyzed data, prepared the figures and drafted the manuscript. HT edited and revised the manuscript for intellectual content, grammar and spelling. All authors approved the final version of the manuscript.

\section{Ethics approval and consent to participate}

Each procedure was approved by the Ethics Committee of the Guangzhou Women and Children's Medical Center (Guangzhou, China) and conformed to the Intrapartum Care: Care of Healthy Women and Their Babies During Childbirth guidelines of the National Collaborating Centre for Women's and Children's Health. All of these patients provided written informed consent.

\section{Patients' consent for publication}

Not applicable.

\section{Competing interests}

The authors declare that they have no competing interests.

\section{References}

1. Kosinova M, Stourac P, Adamus M, Seidlova D, Pavlik T, Janku P Krikava I, Mrozek Z, Prochazka M, Klucka J, et al: Rocuronium versus suxamethonium for rapid sequence induction of general anaesthesia for caesarean section: Influence on neonatal outcomes. Int J Obstet Anesth 32: 4-10, 2017.

2. Finnerup NB, Haroutounian S, Kamerman P, Baron R, Bennett DL, Bouhassira D, Cruccu G, Freeman R, Hansson P, Nurmikko T, et al: Neuropathic pain: An updated grading system for research and clinical practice. Pain 157: 1599-1606, 2016

3. Sevelsted A, Stokholm J, Bønnelykke K and Bisgaard H: Cesarean section and chronic immune disorders. Pediatrics 135: e92-e98, 2015.
4. Mueller NT, Whyatt R, Hoepner L, Oberfield S, Dominguez-Bello MG, Widen E, Hassoun A, Perera F and Rundle A: Prenatal exposure to antibiotics, cesarean section and risk of childhood obesity. Int J Obes (Lond) 39: 665-670, 2015.

5. Huang L, Chen Q, Zhao Y, Wang W, Fang F and Bao Y: Is elective cesarean section associated with a higher risk of asthma? A meta-analysis. J Asthma 52: 16-25, 2015.

6. Mylonas I and Friese K: Indications for and risks of elective cesarean section. Dtsch Arztebl Int 112: 489-495, 2015.

7. McDonnell NJ, Paech MJ, Muchatuta NA, Hillyard S and Nathan EA: A randomised double-blind trial of phenylephrine and metaraminol infusions for prevention of hypotension during spinal and combined spinal-epidural anaesthesia for elective caesarean section. Anaesthesia 72: 609-617, 2017.

8. Kristensen K and Henriksen L: Cesarean section and disease associated with immune function. J Allergy Clin Immunol 137: 587-590, 2016.

9. Ngan Kee WD, Lee SW, Ng FF, Tan PE and Khaw KS: Randomized double-blinded comparison of norepinephrine and phenylephrine for maintenance of blood pressure during spinal anesthesia for cesarean delivery. Anesthesiology 122: 736-745, 2015.

10. Puri GD, Mathew PJ, Biswas I, Dutta A, Sood J, Gombar S, Palta S, Tsering M, Gautam PL, Jayant A, et al: A multicenter evaluation of a closed-loop anesthesia delivery system: A randomized controlled trial. Anesth Analg 122: 106-114, 2016.

11. Li C, Li Y, Wang K and Kong X: Comparative evaluation of remifentanil and dexmedetomidine in general anesthesia for cesarean delivery. Med Sci Monit 21: 3806-3813, 2015.

12. Murouchi T, Iwasaki S and Yamakage M: Quadratus lumborum block: Analgesic effects and chronological ropivacaine concentrations after laparoscopic surgery. Reg Anesth Pain Med 41: 146-150, 2016.

13. Kathuria S, Gupta S and Dhawan I: Dexmedetomidine as an adjuvant to ropivacaine in supraclavicular brachial plexus block. Saudi J Anaesth 9: 148-154, 2015.

14. National Collaborating Centre for Women's and Children's Health (UK): Intrapartum care: Care of healthy women and their babies during childbirth. London, RCOG Press, 2007.

15. Tashjian RZ, Hung M, Keener JD, Bowen RC, McAllister J, Chen W, Ebersole G, Granger EK and Chamberlain AM: Determining the minimal clinically important difference for the American Shoulder and Elbow Surgeons score, simple shoulder test, and visual analog scale (VAS) measuring pain after shoulder arthroplasty. J Shoulder Elbow Surg 26: 144-148, 2017.

16. Dong C, Hu L, Liang F and Zhang S: Effects of electro-acupuncture on labor pain management. Arch Gynecol Obstet 291: 531-536, 2015.

17. Makvandi S, Latifnejad Roudsari R, Sadeghi R and Karimi L: Effect of birth ball on labor pain relief: A systematic review and meta-analysis. J Obstet Gynaecol Res 41: 1679-1686, 2015.

18. Li Y, Hu C, Fan Y, Wang $\mathrm{H}$ and $\mathrm{Xu} \mathrm{H}$ : Epidural analgesia with amide local anesthetics, bupivacaine, and ropivacaine in combination with fentanyl for labor pain relief: A meta-analysis. Med Sci Monit 21: 921-928, 2015.

19. Stourac P, Adamus M, Seidlova D, Pavlik T, Janku P, Krikava I, Mrozek Z, Prochazka M, Klucka J, Stoudek R, et al: Low-dose or high-dose rocuronium reversed with neostigmine or sugammadex for cesarean delivery anesthesia: A randomized controlled noninferiority trial of time to tracheal intubation and extubation. Anesth Analg 122: 1536-1545, 2016.

20. Ripollés Melchor J, Espinosa Á, Martínez Hurtado E, Casans Francés R, Navarro Pérez R, Abad Gurumeta A and Calvo Vecino JM: Colloids versus crystalloids in the prevention of hypotension induced by spinal anesthesia in elective cesarean section. A systematic review and meta-analysis. Minerva Anestesiol 81: 1019-1030, 2015.

21. Visser GH: Women are designed to deliver vaginally and not by cesarean section: An obstetrician's view. Neonatology 107: 8-13, 2015.

22. Happel-Parkins A and Azim KA: At pains to consent: A narrative inquiry into women's attempts of natural childbirth. Women Birth 29: 310-320, 2016.

23. Sawhney M, Mehdian H, Kashin B, Ip G, Bent M, Choy J, McPherson $M$ and Bowry R: Pain after unilateral total knee arthroplasty: A prospective randomized controlled trial examining the analgesic effectiveness of a combined adductor canal peripheral nerve block with periarticular infiltration versus adductor canal nerve block alone versus periarticular infiltration alone. Anesth Analg 122: 2040-2046, 2016. 
24. Wang LZ, Liu X, Zhang YF, Hu XX and Zhang XM: Addition of fentanyl to the ultrasound-guided transversus abdominis plane block does not improve analgesia following cesarean delivery. Exp Ther Med 11: 1441-1446, 2016.

25. Jadon A, Dixit S, Kedia SK, Chakraborty S, Agrawal A and Sinha N: Interscalene brachial plexus block for shoulder arthroscopic surgery: Prospective randomised controlled study of effects of $0.5 \%$ ropivacaine and $0.5 \%$ ropivacaine with dexamethasone. Indian J Anaesth 59: 171-776, 2015.
26. Song J, Li L, Yu P, Gao T and Liu K: Preemptive scalp infiltration with $0.5 \%$ ropivacaine and $1 \%$ lidocaine reduces postoperative pain after craniotomy. Acta Neurochir (Wien) 157: 993-998, 2015.

27. Xiao F, Xu WP, Zhang YF, Liu L, Liu X and Wang LZ: The dose-response of intrathecal ropivacaine co-administered with sufentanil for cesarean delivery under combined spinal-epidural anesthesia in patients with scarred uterus. Chin Med J (Engl) 128: 2577-2582, 2015. 